INRA Prod. Anim., 2011, 24 (4), 307-322

\title{
Contraintes et opportunités d'organisation de la sélection dans les filières porcine et avicole
}

B. COUDURIER

INRA, CODIR Agriculture, 147 rue de l'Université, F-75338 Paris, France

Courriel : bernard.coudurier@tours.inra.fr

Les particularités biologiques des porcs et des volailles offrent des possibilités que les sélectionneurs se doivent d'exploiter dans le contexte concurrentiel qui est le leur. Elles ont conduit à un large recours au croisement entre populations et à un type d'organisation pyramidale caractéristique, dont la mise en œuvre conditionne en retour la typologie des opérateurs génétiques eux-mêmes.

Dans les filières porcine et avicole, l'émergence des schémas de sélection et de croisement remonte déjà à plusieurs décennies. Ils se sont rapidement structurés, progressivement concentrés, et paraissent désormais bien établis car reposant sur un modèle technique et économique stable, caractérisé par des évolutions continues mais sans remise en cause de ses fondements. En s'appuyant sur quelques illustrations et statistiques préférentiellement tirées des conditions usuelles de terrain, le présent article se propose d'expliciter ce qui a amené les opérateurs génétiques des filières porcine et avicole à mettre en place des dispositifs assez comparables, par delà les quelques différences liées à des particularités biologiques ou économiques propres à chacune de ces espèces ou filières. Il s'attachera également à montrer comment, par contrecoup, le mode d'organisation pyramidal des schémas de sélection et de croisement porcins et avicoles a progressivement façonné les opérateurs génétiques eux-mêmes.

\section{1 / Particularités biologi- ques et caractères d'in- térêt : quelques repères}

Les caractéristiques comparées de trois espèces représentatives des ruminants et des monogastriques (bovin à titre de référence, porc et poule) sont résumées à grands traits au tableau 1.

La prolificité de l'espèce et la vitesse de renouvellement des générations

Tableau 1. Particularités biologiques et caractères d'intérêt : quelques repères*.

\begin{tabular}{|c|c|c|c|}
\hline$\left(\begin{array}{l}0 \\
+\end{array}\right)$ & Bovin & Porc & Poule / Poulet \\
\hline Age début reproduction & $2-3$ ans & 1 an & 6 mois \\
\hline Nbre de cycles/an & 1 & $2+$ & $1+-($ continu $)$ \\
\hline $\begin{array}{l}\text { Descendants/mère/ } \\
\text { cycle }\end{array}$ & $1-$ & $10+$ & $150+$ \\
\hline $\begin{array}{l}\text { Particularités } \\
\text { physiologiques }\end{array}$ & $\begin{array}{c}\text { Absence } \\
\text { d'anœestrus } \\
\text { de lactation }\end{array}$ & $\begin{array}{l}\text { Anœstrus } \\
\text { de lactation }\end{array}$ & $\begin{array}{c}\text { Incubation }^{1} \text { des œufs } \\
\text { Glandes } \\
\text { utéro-vaginales }\end{array}$ \\
\hline Conduite & $\begin{array}{c}\text { +- saisonnière } \\
\text { (+- mois) }\end{array}$ & $\begin{array}{c}\text { Bande } \\
(+- \text { jours })\end{array}$ & $\begin{array}{l}\text { Lot d'éclosion }{ }^{2} \\
(+- \text { heures })\end{array}$ \\
\hline $\begin{array}{l}\text { Poids naissance ou } \\
\text { éclosion }(\mathrm{kg})\end{array}$ & $40+-$ & $1,5+-$ & $0,05+-$ \\
\hline $\begin{array}{l}\text { Poids d'entrée en } \\
\text { reproduction }(\mathrm{kg})\end{array}$ & $400+-$ & $150+-$ & $1,5+-/ 2,5+-$ \\
\hline
\end{tabular}

\begin{tabular}{|l|c|c|c|}
\hline $\begin{array}{l}\text { Caractères précoces } \\
(\hat{O})\end{array}$ & $\begin{array}{c}\text { Croissance-carcasse } \\
\text { (veau / taurillon) } \\
\mathrm{N}+9 / 18 \text { mois }\end{array}$ & $\begin{array}{c}\text { Croissance-carcasse } \\
\text { (charcutier) } \\
\mathrm{N}+6 \text { mois }\end{array}$ & $\begin{array}{c}\text { Croissance-carcasse } \\
\text { (poulet) } \\
\mathrm{E}+6 / 12 \text { semaines }\end{array}$ \\
\hline $\begin{array}{l}\text { Caractères tardifs } \\
(+ \text { à la 1ère mesure) }\end{array}$ & $\begin{array}{c}\text { Lactation } \\
\mathrm{V}+10 \text { mois }\end{array}$ & $\begin{array}{c}\text { Portée } \\
\mathrm{MB}+1 \text { mois }\end{array}$ & $\begin{array}{c}\text { Ponte } \\
\mathrm{EP}+10+- \text { mois }\end{array}$ \\
\hline
\end{tabular}

\footnotetext{
* Les chiffres mentionnés ne constituent que des ordres de grandeur, compte tenu des disparités existant entre types génétiques, du fait notamment (mais pas exclusivement) de finalités économiques diversifiées : lait ou viande chez les bovins, chair ou ponte chez la poule.

1 incubation artificielle en couvoir.

2 regroupe l'ensemble des œufs pondus pendant un laps de temps de 1 à 2 semaines.

$\mathrm{N}=$ naissance $; \mathrm{E}=$ éclosion.

$V=$ vêlage $; \mathrm{MB}=$ mise bas $; \mathrm{EP}=$ entrée en ponte.
} 
constituent les facteurs les plus déterminants de l'organisation des schémas de sélection. Une rupture apparaît clairement en faveur des monogastriques, tant pour la prolificité (multiplicité de descendants par cycle, nombre de cycles par an) que pour l'âge à l'entrée en reproduction des femelles. Toutefois, entre espèces monogastriques, l'avantage revient nettement aux volailles en termes d'aptitude à générer de nombreux descendants dans un moindre intervalle de temps (le cycle de ponte, dont la durée est ajustée selon la finalité de la lignée, correspondant à une production continue de descendants, contrairement à la production discontinue des mammifères).

Quelques autres particularités biologiques liées à la reproduction méritent également d'être mentionnées, compte tenu de leur impact sur la conduite des cheptels et/ou sur leur structure familiale. Il s'agit d'une part de l'anœstrus de lactation chez la truie, induisant des retours en chaleurs post-sevrage synchronisés et donc des naissances espacées de quelques jours seulement au sein des bandes, ainsi que de l'incubation (artificielle) des œufs chez les volailles, induisant des éclosions espacées de quelques heures seulement au sein des lots d'éclosion, facteurs favorables à la constitution de populations homogènes en termes de maîtrise du milieu, par rapport à la situation des ruminants. D'autre part, chez les volailles, l'existence de glandes utérovaginales stockant les spermatozoïdes pendant une longue durée conduit, lors des reproductions pedigree, à n'accoupler qu'un seul mâle par femelle (pour éviter de s'astreindre à une période intermédiaire dite de virginisation), d'où une structure familiale de type hiérarchique (descendants intra-mère intra-père) caractéristique des schémas de sélection avicole au niveau des lignées pures.
Enfin, les différences considérables de format des animaux ne placent pas les sélectionneurs des différentes espèces sur un pied d'égalité - la filière volaille étant la plus favorisée - eu égard aux effectifs susceptibles d'être détenus, procréés, phénotypés, éliminés ou diffusés à longue distance, comme il sera précisé par la suite.

Des différences existent également entre espèces dans les délais d'obtention des performances mesurées sur les candidats à la reproduction. Dans le cas des performances précoces (de type croissance carcasse, mesurées une seule fois sur les jeunes des deux sexes), le délai d'obtention à compter de l'événement déclencheur (naissance ou éclosion) se réduit significativement et graduellement en passant du bovin à la poule. Ces différences sont encore plus accusées dans le cas des performances tardives (lactation, portée ou ponte) mesurables de façon répétée sur les seules femelles, le premier événement déclencheur (vêlage, mise bas, entrée en ponte) étant de plus en plus précoce et son renouvellement accéléré (du moins entre bovin et monogastriques).

\section{2 / Maximisation de l'effica- cité de la sélection}

La maximisation du progrès génétique annuel a donné lieu à un article théorique déjà ancien (Ollivier 1974) mais dont les principales conclusions restent valables en tendance, les pratiques actuelles étant voisines de celles recommandées.

\section{L'objectif est :}

- de maximiser $R=i \rho \sigma_{A} / t$ (R étant la réponse annuelle à la sélection, $i$ l'intensité de sélection, $t$ l'intervalle entre générations, $\rho$ la corrélation entre la valeur génétique additive et le critère de sélection et $\sigma_{A}$ l'écart-type génétique additif, paramètre sur lequel le sélectionneur ne peut agir puisqu'il n'est fonction que du caractère et de la population),

- pour deux types de caractères, individuels (valeur de $\rho$ fixe) ou répétés (valeur de $\rho$ variable),

- en fonction des particularités biologiques de cinq espèces (poule, lapin, porc, mouton et bovin),

- sachant que, moyennant certaines simplifications, $i / t$ est fonction du taux de renouvellement annuel des reproducteurs, de même que $i \rho / t$ sous certaines hypothèses concernant la structure de la population.

C'est donc la valeur optimale du taux de renouvellement permettant de maximiser la réponse à la sélection qui est déterminée (sachant que dans le cas des bovins, la forme très plate des courbes de réponse relativise quelque peu cette notion d'optimum).

Quand la précision de l'estimation de la valeur génétique $\rho$ est fixe, ce qui est le cas des caractères individuels, $i / t$ est fonction du taux de renouvellement annuel qu'il faut optimiser. L'intensité de sélection ne pouvant être augmentée qu'au prix d'un accroissement de l'intervalle de générations (pour accroître le nombre de candidats) à taille de population constante, il convient de déterminer le meilleur compromis, et ce pour l'ensemble de la population en prenant en compte les deux sexes, puisque dans les populations sélectionnées, l'intensité de sélection et l'âge à la réforme diffèrent entre mâles et femelles, de même que leurs effectifs respectifs. C'est donc le rapport $i_{m}+i_{f} / t_{m}+t_{f}(m=$ mâles ; $f=$ femelles) qui doit être optimisé, y compris au travers de la proportion de reproducteurs à utiliser dans chaque sexe. Les principaux résultats relatifs aux cas des bovins allaitants, des

Tableau 2. Optimisation du progrès génétique annuel pour des caractères individuels ( 9 ) ou répétés et tardifs ( 9 ) d’après Ollivier 1974.

\begin{tabular}{|l|c|c|c|c|c|c|}
\hline \multirow{2}{*}{$\begin{array}{c}\text { Paramètres } \\
\text { populationnels à } \\
\text { l'optim um }\end{array}$} & \multicolumn{2}{|c|}{ caractères individuels (j+) } & \multicolumn{3}{c|}{ caractères répétés (tardifs +) } \\
\cline { 2 - 7 } & $\begin{array}{c}\text { Bovin } \\
\text { allaitant } \\
\text { (V3 ans) }\end{array}$ & $\begin{array}{c}\text { Porc } \\
\text { (charcutier) }\end{array}$ & $\begin{array}{c}\text { Poulet } \\
\text { (chair) }\end{array}$ & $\begin{array}{c}\text { Bovin } \\
\text { laitier } \\
\text { (V2 ans) }\end{array}$ & $\begin{array}{c}\text { Porc } \\
\text { (prolificité) }\end{array}$ & $\begin{array}{c}\text { Poule } \\
\text { (ponte) }\end{array}$ \\
\hline Ratio \% / & 20 & 15 & 10 & - & - & - \\
\hline Max. réponse / an (en $\sigma_{A}$ ) & 0,33 & 1,75 & 2,89 & 0,15 & 0,35 & 1,10 \\
\hline $\begin{array}{l}\text { Taux de renouvellement } \\
\text { annuel }(\%)\end{array}$ & 26 & 100 & 128 & $40^{*}$ & 96 & 123 \\
\hline $\begin{array}{l}\text { Taux de renouvellement } \\
\text { annuel \% (\%) }\end{array}$ & 20 & 92 & 122 & 24 & 69 & 120 \\
\hline Age réforme (années) & 6,9 & 1,2 & 1,0 & 6,3 & 1,9 & 1,0 \\
\hline
\end{tabular}

$V=$ vêlage .

* Hors testage sur descendance, traité à part (conduisant à une réponse du même ordre de grandeur, avec allongement de l'âge à la réforme et réduction du taux de renouvellement). 
Tableau 3. Importance moyenne des effets d'hétérosis direct $(H)$ et héritabilité $\left(h^{2}\right)$ par catégorie de caractères*, d'après Sellier 2010.

\begin{tabular}{|l|c|c|}
\hline \multicolumn{1}{|c|}{ Catégorie de caractères } & $\begin{array}{c}\mathrm{H} \\
\mathbf{( \% )}\end{array}$ & $\begin{array}{c}\mathrm{h}^{2} \\
\mathbf{( \% )}\end{array}$ \\
\hline $\begin{array}{l}\text { Fertilité q, prolificité, taux de ponte, survie du } \\
\text { jeune, longévité fonctionnelle des mères }\end{array}$ & $10-15$ & $3-15$ \\
\hline $\begin{array}{l}\text { Vitesse de croissance post-sevrage, production } \\
\text { laitière, précocité sexuelle (mammifères), âge } \\
\text { au 1 }{ }^{\text {er }} \text { cuf, efficacité alimentaire en croissance }\end{array}$ & $5-10$ & $20-35$ \\
\hline $\begin{array}{l}\text { Morphologie, composition du produit (carcasse, } \\
\text { qualité de la viande, matières utiles du lait) }\end{array}$ & $0-2$ & $40-60$ \\
\hline
\end{tabular}

* Hétérosis observée lors de croisements entre races usuelles. porcs charcutiers et des poulets de chair figurent au tableau 2.

Quand la mesure du caractère peut être répétée dans le temps, un gain de précision de l'estimation de la valeur génétique $\rho$ peut être obtenu au prix d'un allongement de l'intervalle entre générations. La réponse maximale à la sélection est alors fonction de $i \rho / t$ (et non plus seulement de $i / t$ ). Le ratio peut néanmoins être maximisé, en le réduisant également à une fonction de la durée de vie moyenne des reproducteurs dans une population supposée d'effectif stable et de taille suffisante pour que son effet sur l'intensité de sélection puisse être ignoré. Comme dans le cas précédent, il doit prendre en compte les deux sexes, bien que les performances ne soient mesurées que chez les femelles. Les principaux résultats relatifs aux cas de la lactation chez la vache, de la prolificité chez la truie et de la ponte chez la poule figurent au tableau 2 .

Pour l'essentiel, ces résultats conduisaient à recommander un renouvellement des reproducteurs d'autant plus rapide que l'espèce est prolifique, et ce dans la limite du délai nécessaire à l'obtention d'un nombre suffisant de performances répétées. Ces recommandations impliquent toutefois que l'effectif génétique des populations soit assez grand pour limiter l'accroissement du taux de consanguinité qui en résulte. Elles restent encore d'actualité : les ratios femelles/mâle, taux de renouvellement des reproducteurs (plus élevés chez les mâles) et âges à la réforme constatés chez les sélectionneurs sont effectivement du même ordre de grandeur, du moins en filières porcine et avicole (dans le cas des bovins laitiers, le recours massif au testage sur la descendance conjugué à une dégradation de la fertilité dans certaines races a conduit à des écarts significatifs par rapport aux données rapportées au tableau 2). Ces résultats montraient par ailleurs qu'en matière de réponse annuelle à la sélection, les monogastriques sont nettement avantagés par rapport au bovin du fait de leur caractéristiques biologiques, la poule en particulier.

\section{3 / Utilisation du croisement pour exploiter l'hétérosis}

Du fait de leur nombre élevé de produits femelles par mère et par an (ou taux de reproduction), les porcs et les volailles se prêtent particulièrement bien au croisement. Cette pratique permet de bénéficier du phénomène d'hétérosis, classiquement défini chez les animaux comme l'écart entre le produit de croisement et la moyenne des populations parentales. Cet effet s'exerce à la fois sur la composante directe (individu $\mathrm{F}_{1}$ ) et sur la composante maternelle (mère $\mathrm{F}_{1}$ ), voire sur la composante paternelle (père $F_{1}$ ). Le modèle génétique sous-jacent repose majoritairement sur la dominance, à l'origine de la plus grande part de la variance génétique non additive symbolisée par $\mathrm{H}$, en complément du modèle additif basé sur l'effet moyen de substitution allélique, dont la part de variance correspond à l'héritabilité $h^{2}$. L'ampleur de l'effet d'hétérosis est fortement conditionnée par la nature des caractères, comme rapporté au tableau 3 pour l'effet d'hétérosis direct (Sellier 2010). Elle est maximale pour les caractères de type «fitness», liés à la fécondité et à la survie, et s'estompe avec l'âge pour les autres types de caractères, jusqu'à devenir quasi nulle pour les caractères de morphologie, de composition corporelle relation inverse est observée entre hétérosis (effets non-additifs des gènes) et héritabilité (effets additifs). L'hétérozygotie générée par le croisement présente donc l'avantage d'améliorer des caractères de forte importance économique mais à héritabilité faible, d'autant que les effets d'hétérosis directs et maternels peuvent se cumuler pour des caractères complexes mettant en jeu plusieurs fonctions biologiques, comme le poids de portée sevrée par truie mise à la reproduction. L'accroissement d'hétérozygotie exerce en outre un effet tampon face aux variations accidentelles (ou permanentes) des conditions de milieu, conduisant à une moindre sensibilité aux variations environnementales, donc in fine à une plus grande homogénéité des performances (Sellier 2010).

L'hétérosis dépend fortement de la distance génétique entre les populations parentales, comme illustré au tableau 4 (Bidanel 2011) dans le cas du porc pour la taille de portée au sevrage. Pour la composante maternelle en particulier, les croisements entre les races Large White et Meishan se révèlent nettement plus «hétérotiques» que ceux pratiqués entre races européennes et/ou américaines, du fait de la grande distance génétique observée entre races chinoises et races européennes (ou américaines). La taille de portée au sevrage étant un critère d'importance économique majeure (elle explique $50 \%$ environ des variations de rentabilité d'un élevage naisseur-engraisseur), le recours à des truies de production $\mathrm{F}_{1}$ est rapidement devenu la règle. A titre d'illustration, l'effet d'hétérosis maternel procuré par le croisement classique entre races (ou variétés de races) Large White et Landrace Français est de l'ordre de 0,6 porcelet sevré, soit $+7 \%$ par rapport à la moyenne des races parentales (Legault 1998). Chez le porc en particulier, la création de nombreuses lignées composites, tant en voie mâle que femelle (incluant parfois dans ce dernier cas la race Meishan), constitue une autre forme d'exploitation «à la marge» de l'effet d'hétérosis, ces lignées composites permettant le maintien d'une fraction de l'hétérosis initial. Toutefois, l'objectif et la qualité des produits. En outre, une premier reste avant tout de réunir dans

Tableau 4. Exemples d'hétérosis chez le porc, d'après Bidanel 2011.

\begin{tabular}{|l|c|c|c|c|c|c|}
\hline \multirow{2}{*}{$\begin{array}{l}\text { Hétérosis sur la taille } \\
\text { de portée au sevrage }\end{array}$} & \multicolumn{3}{|c|}{$\begin{array}{c}\text { Entre races européennes } \\
\text { et/ou américaines }\end{array}$} & \multicolumn{3}{|c|}{$\begin{array}{c}\text { Entre Large White } \\
\text { et Meishan }\end{array}$} \\
\cline { 2 - 8 } & Unités & $\%$ & $\mathrm{~N}$ & Unités & $\%$ & $\mathrm{~N}$ \\
\hline Effet maternel (truie) & 0,84 & 9 & 9 & 2,43 & 23 & 3 \\
\hline Effet direct (portée) & 0,49 & 6 & 16 & 0,67 & 6 & 3 \\
\hline
\end{tabular}

Unités $=$ en unité physique du caractère.

$\%=$ en pourcentage de la moyenne des races parentales.

$\mathrm{N}=$ nombre d'estimations. 
Figure 1. Effet d'hétérosis chez le canard mulard, d'après Baéza et al 2004.

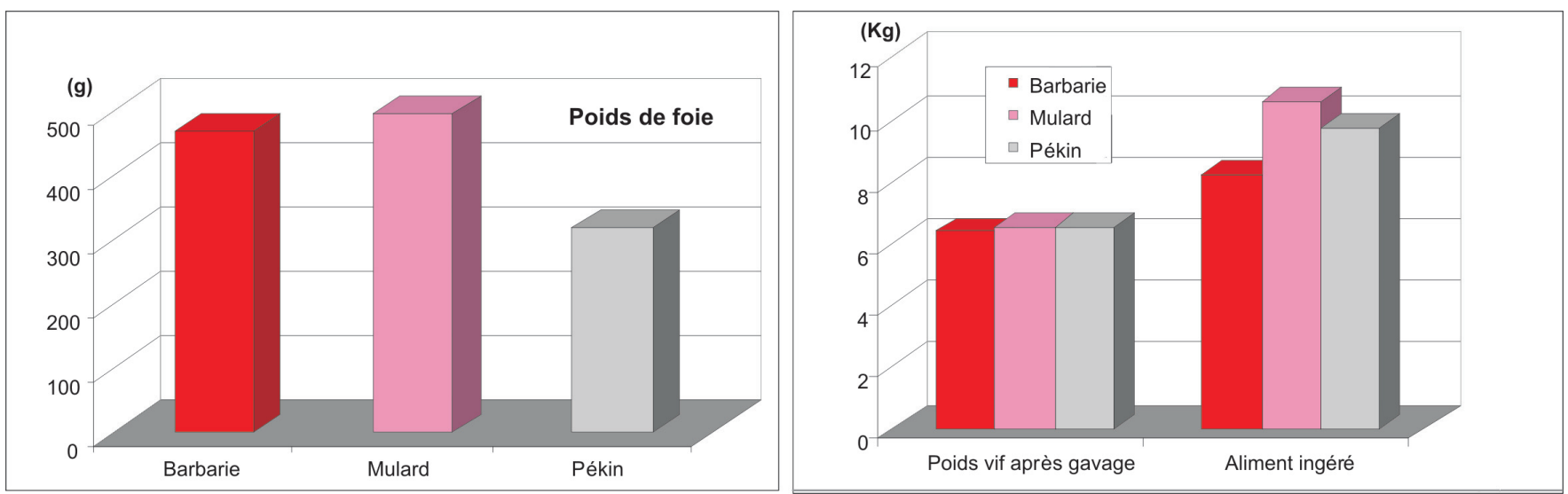

une même lignée des races jugées complémentaires.

Le croisement canard de Barbarie $\mathrm{x}$ cane Pékin, conduisant à la production d'un hybride inter-générique, le canard mulard, fournit un exemple assez rare de sur-dominance, illustré à la figure 1 (Baéza et al 2004). A poids vif quasi égal en fin de gavage, l'hybride présente en effet un léger surcroît de poids de foie par rapport au meilleur génotype parental (+ 4\% par rapport au Barbarie). Il s'explique en grande partie par la combinaison d'aptitudes parentales contrastées : l'orientation préférentielle du métabolisme lipidique vers la stéatose hépatique chez le père Barbarie et la capacité d'ingestion chez la mère Pékin (dont la quantité d'aliment ingéré dans un contexte de gavage constitue un indicateur).

\section{4 / Autres avantages de la combinaison de races et/ou lignées au sein d'un plan de croisement}

\section{1 / Optimisation de la complé- mentarité entre populations par- ticipant au plan de croisement}

L'effet de complémentarité entre races et/ou lignées constitue un autre argument majeur en faveur du croisement. Il réside dans la possibilité de combiner des populations aux aptitudes contrastées, leur complémentarité étant raisonnée en fonction de la place que chacune sera amenée à occuper dans le plan de croisement (voie mâle ou voie femelle). Cet effet est exploitable indépendamment d'éventuels effets d'hétérosis.

a) Chercher à s'affranchir de liaisons génétiques défavorables entre groupes de caractères

Chez les espèces de rente, l'antagonisme génétique le plus constant est observé entre les caractères dits de reproduction (exprimés pour l'essentiel chez la mère) et les caractères dits de production (exprimés chez le produit). Or, chez les espèces à taux de reproduction élevé, l'importance économique du coût de production du jeune conduit à accorder autant d'attention à l'efficacité des reproductrices qu'aux aptitudes du produit terminal (chair ou pondeuse). L'ampleur de cet antagonisme peut néanmoins varier selon les caractères considérés, l'espèce, ainsi que les races ou lignées intra-espèce (lesquelles évoluent par ailleurs dans le temps), comme illustré dans les deux exemples qui suivent.

Chez le porc, des corrélations génétiques entre caractères de production et

Tableau 5. Exemple de corrélations génétiques entre caractères de production et prolificité chez le porc, d'après Tribout et Bidanel 2008.

\begin{tabular}{|c|l|c|}
\hline $\begin{array}{c}\text { Caractère de } \\
\text { production }\end{array}$ & \multicolumn{1}{|c|}{ Race } & $\begin{array}{c}\text { corrélation génétique avec le nombre } \\
\text { de nés vivants (portées 1 à 3)* }\end{array}$ \\
\hline \multirow{2}{*}{ Age à $100 \mathrm{~kg}$} & $\begin{array}{l}\text { Large White } \\
\text { lignée femelle }\end{array}$ & $0,28 / 0,31$ \\
\cline { 2 - 3 } & Landrace Français & $0,28 / 0,32$ \\
\hline \multirow{2}{*}{ Lard à $100 \mathrm{~kg}$} & $\begin{array}{l}\text { Large White } \\
\text { lignée femelle }\end{array}$ & $0,09 / 0,20$ \\
\cline { 2 - 3 } & Landrace Français & $0,09 / 0,16$ \\
\hline
\end{tabular}

* Chaque rang de portée a été considéré indépendamment (et non comme un caractère répété). prolificité (nombre de nés vifs lors des 3 premières portées) sont présentées au tableau 5 pour deux races utilisées en voie femelle (Tribout et Bidanel 2008). Ces corrélations sont globalement défavorables, mais restent modérées (pour l'âge à $100 \mathrm{~kg}$ ) ou faibles quoique variables selon le rang de portée (pour l'épaisseur de lard à $100 \mathrm{~kg}$ ), et ce dans les deux races. Des corrélations globalement plus faibles (en particulier chez le Landrace Français) avaient toutefois été estimées douze ans plus tôt dans ces mêmes populations (Ducos et Bidanel 1996), ce qui suggère une probable évolution génétique au cours de la période.

Chez la dinde, des corrélations génétiques entre caractères de croissance et de ponte, mesurée lors de deux périodes consécutives, sont présentées au tableau 6 pour deux lignées utilisées en voie femelle (Chapuis et al 1996). L'ampleur de ces corrélations globalement défavorables varie considérablement selon la période de ponte ou la lignée.

On notera que les corrélations génétiques présentées aux tableaux 5 et 6 ont été estimées dans des populations contribuant à la voie femelle des plans de croisement, les deux types de caractères n'étant mesurés en routine que dans ces populations. Cet état de fait résulte de la spécialisation des populations entre les voies mâle et femelle, laquelle permet de s'affranchir totalement de l'antagonisme génétique entre caractères de production et de reproduction au stade du croisement terminal. Les lignées concourant à la voie mâle ne sont effectivement sélectionnées que pour les seuls caractères de production, dans les limites du maintien de la fertilité mâle.

$\mathrm{Au}$ sein des populations concourant à la voie femelle, par contre, il faut composer avec l'antagonisme génétique entre types de caractères, la voie femelle 
Tableau 6. Exemple de corrélations génétiques entre caractères de croissance et de ponte chez la dinde, d'après Chapuis et al 1996.

\begin{tabular}{|c|c|c|c|}
\hline \multirow[b]{2}{*}{ Caractère de production } & \multirow{2}{*}{$\begin{array}{l}\text { Lignée } \\
\text { (voie } \\
\text { femelle) }\end{array}$} & \multicolumn{2}{|c|}{$\begin{array}{l}\text { Corrélation génétique avec } \\
\text { les caractères de reproduction }\end{array}$} \\
\hline & & $\begin{array}{c}\text { Nombre } \\
\text { d'œufs début } \\
\text { de ponte }\end{array}$ & $\begin{array}{l}\text { Nombre d'œufs } \\
\text { persistance }\end{array}$ \\
\hline \multirow{2}{*}{ Poids à 16 semaines f* $^{*}$} & A & $-0,51$ & $-0,46$ \\
\hline & B & $-0,11$ & $-0,38$ \\
\hline \multirow{2}{*}{ Poids à 16 semaines ${ }^{*}$} & A & $-0,37$ & $-0,38$ \\
\hline & $\mathrm{B}$ & $-0,17$ & $-0,31$ \\
\hline
\end{tabular}

* Caractères considérés comme indépendants compte tenu de l'ampleur du dimorphisme sexuel.

contribuant in fine à la moitié du patrimoine génétique des produits terminaux pour les caractères de production. L'indice global de sélection constitue donc un compromis entre un objectif prioritaire relatif aux critères de reproduction, lesquels bénéficient d'une forte pondération, et des objectifs secondaires relatifs aux critères de production, affectés d'une pondération moindre. Cette démarche est illustrée à la figure 2 dans le cas de la race porcine Landrace Français (Delaunay, communication personnelle). Le progrès génétique obtenu pour les différents caractères est alors fonction de ces pondérations, mais également de l'ampleur des antagonismes éventuels entre caractères. Comme illustré dans les deux exemples précédents, ces antagonismes sont susceptibles de varier fortement d'une population à l'autre, voire dans le temps pour les mêmes populations. Les stratégies de pondération voire d'évaluation génétique doivent donc être adaptées, le cas échéant. Dans le cas du porc, les corrélations génétiques estimées à l'époque de la mise en place du BLUP modèle animal (Ducos et Bidanel 1996) étaient suffisamment faibles pour justifier des évaluations génétiques indépendantes des deux types de caractères. Ce choix n'a pas été remis en cause au vu des résultats plus récents (Tribout et Bidanel 2008), les valeurs génétiques estimées à partir d'évaluations génétiques indépendantes restant très proches de celles obtenues à partir d'une évaluation conjointe, beaucoup plus lourde. Dans le cas de la dinde par contre, l'ampleur des antagonismes génétiques entre caractères a nécessité d'emblée le recours à une évaluation génétique conjointe pour l'ensemble des caractères (Chapuis et al 1996).

Toutefois, des antagonismes génétiques plus ou moins importants sont également observés entre caractères de production comme entre caractères de reproduction. Citons à titre d'illustration, chez le porc, les corrélations légèrement défavorables entre vitesse de croissance et adiposité de la carcasse
(Bidanel et al 1994, Ducos et Bidanel 1996), ou celles beaucoup plus marquées entre caractères de qualité de la viande et caractères de croissance, efficacité alimentaire et composition corporelle, la teneur en muscles en particulier (Tribout et al 1996). Chez la poule pondeuse, où l'œuf, caractère de reproduction, constitue de fait le produit, les corrélations génétiques obtenues dans deux lignées (Besbes et al 1992) montrent un antagonisme plutôt modéré entre le nombre d'œufs pondus et leur densité (un indicateur de solidité de la coquille) d'une part, et le poids moyen des œufs d'autre part. L'antagonisme le plus marqué était observé entre le poids d'œuf et la densité, mais restait modéré.

La gestion de ces antagonismes intracaractères de production ou de reproduction ne donne pas lieu à une stratégie universelle, comme dans le cas de l'opposition reproduction-production. Selon le cas, l'accent sera mis sur la relative complémentarité des populations combinées dans le plan de croisement ou sur le recours à un indice global de sélection assurant un compromis de même type au sein de chacune des populations utilisées.

b) Maximiser le progrès génétique annuel pour les caractères d'intérêt prioritaires selon l'orientation de la race ou lignée

La spécialisation des populations offre également l'opportunité de maximiser le progrès génétique pour les caractères prioritaires en fonction de l'orientation de la race ou lignée. Un exemple particulièrement illustratif est fourni par la scission, au cours des années 90, de la population Large White des Livres Généalogiques Porcins Collectifs (LGPC) en deux rameaux distincts de type femelle et mâle. A la suite des travaux menés sur les lignées dîtes hyperprolifiques dès les années 70 (Legault 1998), les objectifs de sélection de cette population accordaient une large part à l'amélioration de la prolificité. C'est donc un rameau de type mâle, destiné à la production de verrats terminaux (en croisement avec le Piétrain), qui a été progressivement constitué, par criblage au sein de la population des animaux les plus aptes à en devenir les fondateurs. Les premiers résultats différentiels (Tribout et al 1998) ont été régulièrement actualisés. L'évolution du niveau génétique dans les deux lignées est représentée à la figure 3 (IFIP 2008, Tribout, communication personnelle). A noter que du fait d'un pas de temps différent entre bases mobiles utilisées lors des évaluations reproduction et production, la période représentée est différente.

Figure 2. Exemple de pondération par grande catégorie de caractères chez une race porcine de la voie femelle, le Landrace Français, d'après Delaunay, communication personnelle.

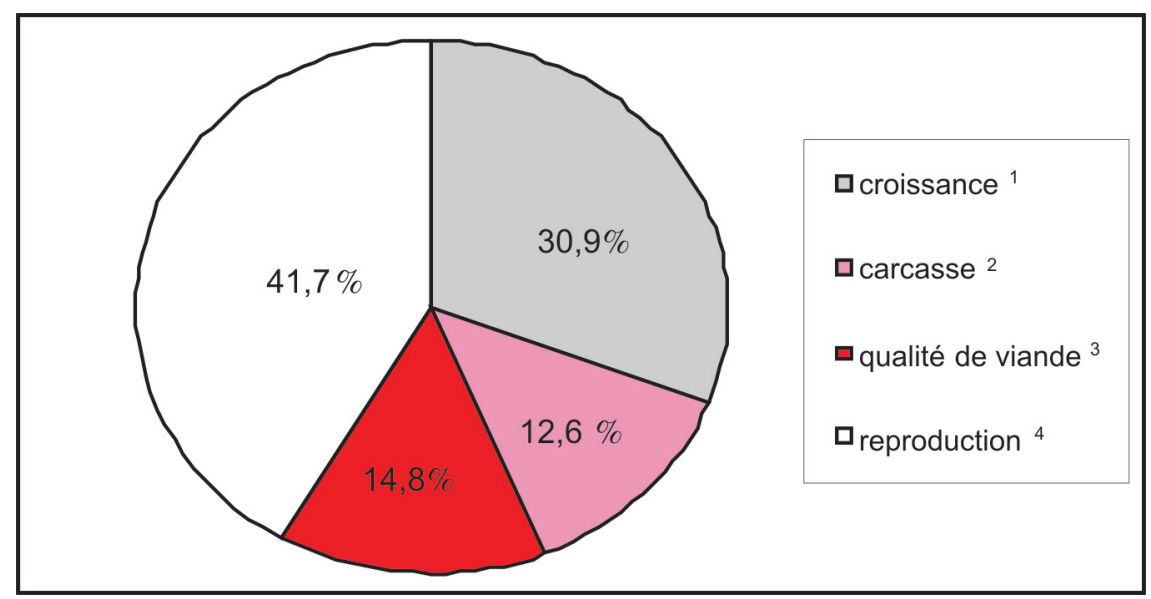

Indice global en vigueur depuis 2010 :

1 gain moyen quotidien, indice de consommation, consommation moyenne journalière ;

2 taux de muscle, rendement de carcasse ;

3 indice composite ;

4 nombre de nés vivants, de sevrés, de tétines. 
Figure 3. Exemples d'évolution génétique selon l'orientation des populations chez le porc Large White : évolution depuis 1994 (a) ou 2004 (b) de la valeur génétique moyenne* de chacun des rameaux voie femelle et voie mâle pour le nombre de nés vivants (a), l'âge à $100 \mathrm{~kg}$ et l'épaisseur de lard à 100 kg (b), d'après IFIP 2008 et Tribout, communication personnelle.
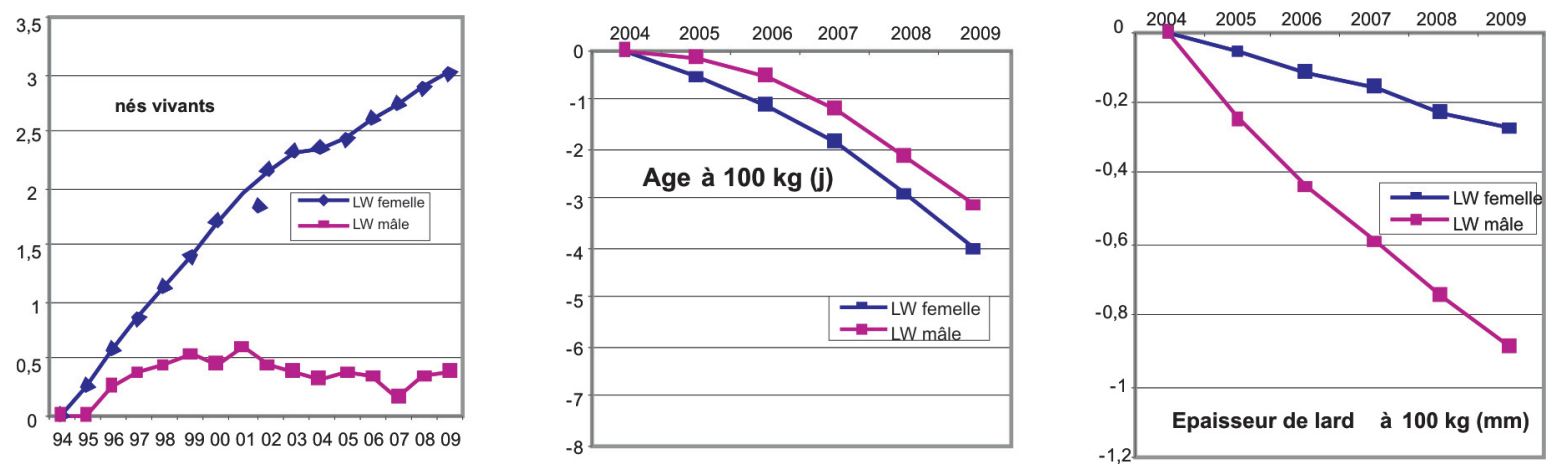

* Valeurs génétiques moyennes rendues artificiellement égales pour la première année de chaque courbe, permettant de comparer les évolutions mais pas les valeurs absolues.

\section{2 / Utilisation de gènes majeurs}

La disponibilité de plusieurs populations à combiner dans un même plan de croisement permet également de tirer parti de certains gènes individuels à effet important sur un ou plusieurs caractères, dans le cas particulier où le mode d'action de ces gènes n'est pas strictement additif pour l'ensemble des caractères qu'ils gouvernent (une simple sélection intra-population étant suffisante dans le cas contraire). L'avantage, dans le cas des gènes majeurs, est de disposer d'une connaissance précise des phénotypes associés aux différents génotypes possibles (intégrant à la fois les effets génétiques additifs et non-additifs), et ce pour les différents caractères influencés par le gène. Les phénotypes résultants présentant généralement des avantages et des inconvénients, le génotype associé aux phénotypes globalement les plus avantageux (en termes de bilan net) peut dès lors être produit via le plan de croisement, en combinant des races et/ou lignées dans lesquelles les allèles à assortir ont été préalablement fixés (ou éradiqués) par sélection.

L'utilisation du gène de la sensibilité à l'halothane (Hal) chez le porc constitue un premier exemple (Sellier 1998). Ce gène autosomal est pléiotrope, l'allèle $n$ (muté) étant récessif pour le syndrome d'hyperthermie maligne, quasi récessif pour le syndrome de viandes pâles et exsudatives (PSE) et additif pour l'hypermuscularité, laquelle confère un avantage substantiel de teneur en viande maigre aux animaux porteurs. Pour permettre la mise en œuvre du plan de croisement décrit à la figure 4, l'allèle $\mathrm{n}$ a été préalablement éradiqué dans les populations de la voie femelle autrefois porteuses (Landrace Français notamment) et fixé (ou maintenu dans cet état) dans celles de la voie mâle.
Cette combinaison conduit à des animaux hétérozygotes qui ne sont pas prédisposés au syndrome de stress et peu prédisposés au syndrome PSE, tout en bénéficiant d'un avantage pour l'hypermuscularité.

Un autre exemple particulièrement emblématique est celui de l'utilisation $\mathrm{du}$ gène de nanisme ( $\mathrm{dw}$ pour $d$ warf) chez la poule (Mérat 1984). Ce gène, lié au sexe, est présent sur le chromosome $\mathrm{Z}$ dont les femelles ne portent qu'une seule copie, le déterminisme du sexe étant du type ZW chez la poule. L'allèle muté (dw) est récessif et diminue d'environ $30 \%$ le poids adulte des reproductrices porteuses, entraînant une réduction de 20 à $25 \%$ des besoins ali- mentaires, assimilable à un rationnement spontané et favorable en termes de bien-être animal. Le coût de production des poussins d'un jour s'en trouve abaissé d'environ $15 \%$, du fait des économies de nourriture et d'espace associées à une souche naine. Pour permettre la mise en œuvre du plan de croisement décrit à la figure 5, l'allèle dw a été fixé dans des lignées de la voie femelle (Vedette INRA), après une série de croisements permettant de minimiser notamment les effets réducteurs de l'allèle dw sur le poids de l'œuf. Les lignées utilisées en voie mâle sont, quant à elles, de taille normale. La combinaison de femelles naines $(\mathrm{dw} /-)$ et de mâles normaux (Dw/Dw) permet de produire des poulets de chair à croissance norma-

Figure 4. Exemple d'utilisation du gène majeur Hal (halothane) chez le porc.

\section{Plan de croisement ( $\mathrm{n}=$ allèle muté)}

( $\mathrm{F} / \mathrm{M}=$ voie femelle / mâle)

\begin{tabular}{|c|c|}
\hline $\begin{array}{c}\text { Repro F } \\
\text { NN }\end{array}$ & $\begin{array}{l}\text { x Repro M } \\
\text { nn } \\
\text { Nn }\end{array}$ \\
\hline Syndrome de stress & $\Rightarrow$ résistant ( $\mathrm{n}$ récessif) \\
\hline $\begin{array}{l}\text { Syndrome PSE } \\
\text { Hypermuscularité }\end{array}$ & $\begin{array}{l}\Rightarrow \text { normal }(\mathrm{n} \pm \text { récessif }) \\
\Rightarrow \text { additif }\end{array}$ \\
\hline
\end{tabular}

Figure 5. Exemple d'utilisation du gène majeur dw (nanisme lié au sexe) chez la poule.

Plan de croisement (dw = allèle muté)

( $\mathrm{F} / \mathrm{M}=$ voie femelle / mâle)

$\begin{array}{cl}\begin{array}{c}\text { Repro } F \\ \text { dw / - }\end{array} & \begin{array}{l}\text { Repro } \\ \text { Dw / Dw }\end{array}\end{array}$

Dw $/$ - $\Rightarrow$ normale (sexe hétérogamétique)

Dw / dw $\Rightarrow$ normal (dw récessif) 
le (chez les femelles Dw/-) ou quasi normale (- 2 à $3 \%$ chez les mâles Dw/dw).

De nombreux autres gènes individuels sont également utilisés en sélection avicole à différentes fins, le plus souvent dans le cadre de croisements. On peut citer à titre d'exemple chez la poule le gène K (pour Kurzer Flugel), gène d'emplumement lent lié au sexe, permettant de distinguer à l'éclosion les mâles des femelles, ainsi que le gène $\mathrm{Na}$ (pour naked neck), induisant une très forte réduction des zones emplumées, incluant notamment le cou, associé à une thermotolérance améliorée et généralement utilisé (dans les souches Label Rouge) à l'état hétérozygote du fait de son additivité. De nombreux gènes de couleur font par ailleurs l'objet d'une large utilisation. Dans la filière chair standard où les poulets à plumage blanc sont la règle, le gène dominant I (pour inhibitor of black) est fixé dans les lignées, permettant d'inhiber la formation des pigments noir et brun (eumélanines) et, dans une moindre mesure, celle des pigments jaune à rouge (phæomélanines), d'où sa dénomination courante et quelque peu abusive de blanc dominant. Quand la coloration rouge doré est par ailleurs présente dans certaines des lignées à combiner, l'inhibition des phæomélanines est obtenue par croisement en mettant en jeu l'allèle $S$ (pour silver), lié au sexe. La fixation dans les lignées de l'allèle c (pour non colored), appelé blanc récessif, constitue une alternative puisqu'il inhibe toute coloration du plumage. A l'opposé, en filière Label Rouge, des colora-

Figure 6. Les schémas de sélection et de multiplication porcine au 31/12/2007 en France, d'après Agence de la Sélection Porcine 2011.

\section{Voie mâle Voie femelle}

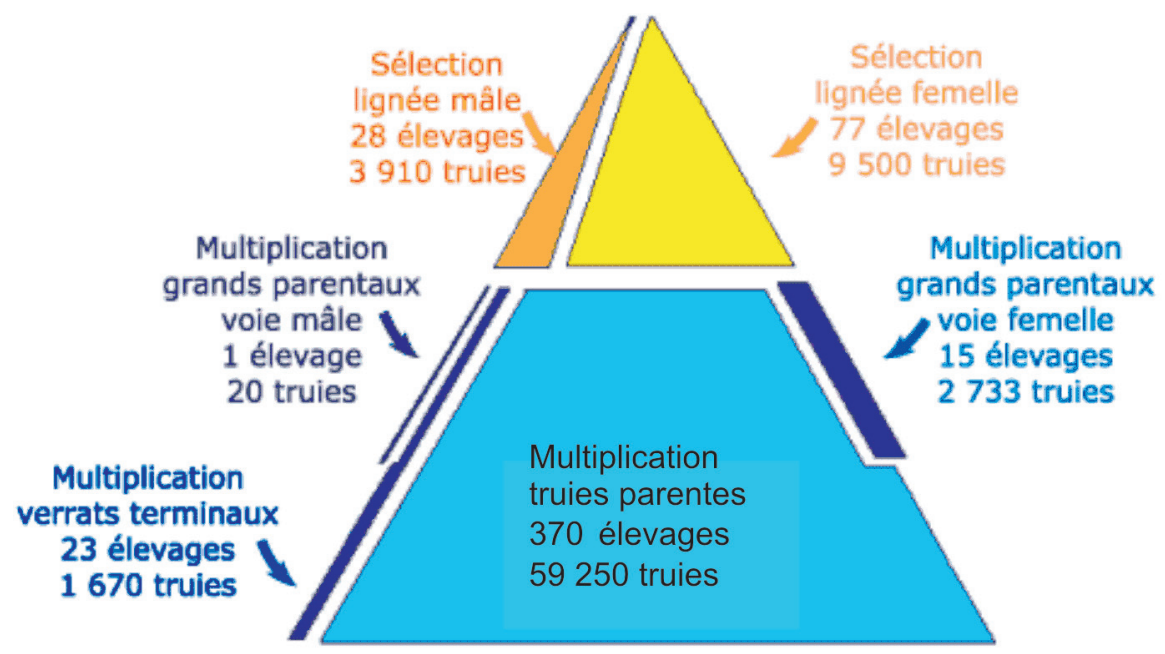

\section{5 / Organisation des sché- mas de sélection et de croi- sement}

\section{1 / La sélection porcine en France... et dans le monde}

Par rapport à d'autres pays, le dispositif français de sélection multiplication porcine est connu d'une manière relativement précise. Cette connaissance a même été quasi exhaustive jusqu'à une période récente, au travers d'enquêtes annuelles diligentées par l'Agence de la Sélection Porcine sur les activités des Organisations de Sélection Porcine (OSP) agréés. Après l'assouplissement de certaines dispositions d'agrément en application de la Loi d'Orientation Agricole de 2006, certaines données ne sont plus communiquées de façon régulière pour la totalité des types génétiques. L'état du dispositif illustré à la figure 6 concerne l'exercice 2007 (Agence de la Sélection Porcine 2011). En voie femelle, la production de truies parentales résulte systématiquement du croisement de deux, voire trois, populations (avec dans ce cas un étage de production intermédiaire de reproductrices grand parentales). La diffusion des deux sexes, permettant de valoriser les animaux produits via des croisements réciproques, est la règle. Au sein de la voie femelle, les effectifs de truies en sélection, en multiplication (y compris de grand parentales) et en production (stricto sensu, d'après IFIP 2008) sont respectivement de 9500, 62000 et 1157000 , soit un rapport d'effectifs détenus pour «descendre» d'un étage à l'autre de x 6,5 et x 18,7 respectivement. En voie mâle en revanche, les effectifs détenus en sélection sont très supérieurs à ceux de l'étage multiplication (la production intermédiaire de reproducteurs grand parentaux y étant anecdotique). Cette situation paradoxale résulte du recours de plus en plus important à des verrats terminaux issus de populations pures. Ainsi, en 2007, le Piétrain de race ou lignées pures représentait plus de la moitié des verrats détenus dans les centres d'IA et presque les deux tiers des doses diffusées (IFIP 2008).

A l'étage de sélection, le dispositif collectif (fédéré par les LGPC) est prépondérant, puisqu'il représentait plus de la moitié des effectifs de truies appartenant à quatre races seulement, comme illustré à la figure 7 pour l'exercice 2007 (Agence de la Sélection Porcine 2011). Ce dispositif coexiste avec celui de la sélection autonome, caractérisé par un grand nombre de variété de races ou de lignées composites, aux effectifs généralement plus modestes. Elles sont majoritairement détenues par des opéra- 
Figure 7. Répartition des effectifs de truies en sélection au 31/12/2007 en France, par catégorie de type génétique, d'après Agence de la Sélection Porcine 2011.

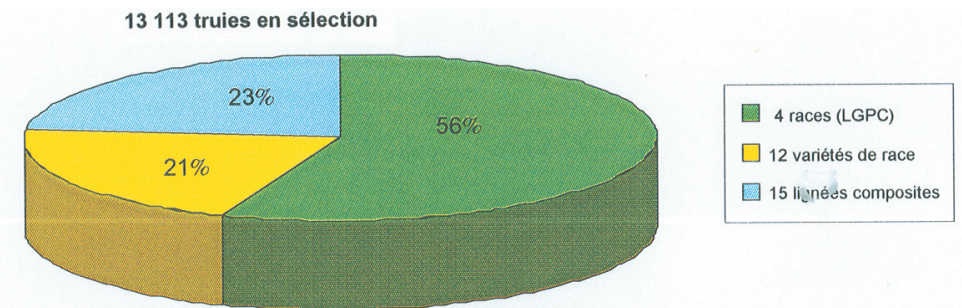

LGPC = Livres Généalogiques Porcins Collectifs, en charge de l'orientation des races Large White lignée femelle, Landrace Français, Large White lignée mâle et Piétrain

teurs privés (nationaux et internationaux), mais parfois également par des acteurs du dispositif collectif, dans un but de diversification de leurs populations en sélection.

Cette dualité disparaît à l'étage de multiplication, une très grande variété de types génétiques étant proposée par les opérateurs comme le montre la consultation du site de l'Agence de la Sélection Porcine (http://www.asp. asso.fr). Ils résultent de la combinaison de nombreuses populations à la disposition des sélectionneurs, dans le cadre collectif et/ou autonome. La concurrence $\mathrm{y}$ est la règle, $\mathrm{y}$ compris entre types génétiques issus d'une même combinaison de populations relevant du dispositif collectif.

Dans le cadre du dispositif d'agrément des OSP, certains résultats obtenus en conditions comparatives standardisées sont par ailleurs disponibles. Le test de contrôle des produits terminaux (porcs charcutiers) est réalisé en continu depuis près de 40 ans, le dernier test publié étant le 29ème (IFIP 2009). Une agrégation, par OSP agréée, des données de la Gestion Technique des Troupeaux de Truies parentales est également réalisée, mais ne donne lieu qu'à une publication anonymée (Agence de la Sélection Porcine 2011).

La coexistence entre un dispositif ouvert, reposant fréquemment sur un fondement coopératif national, et un dispositif autonome, aux mains d'opérateurs privés, tant nationaux qu'internationaux, est également observée dans d'autres pays, en particulier en Europe. Il existe toutefois de fortes variations de la part respective de l'un et l'autre secteur. Globalement, les parts de marché détenues par les opérateurs nationaux restent généralement prépondérantes, qu'ils relèvent de l'un ou l'autre secteur. En d'autres termes, les grands opérateurs internationaux de la sélection porcine se partagent une partie du marché mondial mais ne sont pas à proprement parler en situation dominante.

\section{2 / La sélection avicole mon- diale... et la situation française}

L'univers de la sélection avicole est dominé par quelques très grands groupes (souvent d'origine familiale) diffusant une gamme relativement limitée de produits commerciaux adaptés à la demande mondiale de masse. La concentration peut y être extrême : l'essentiel du marché des reproducteurs standard est à ce jour couvert par deux sélectionneurs de poules pondeuses et deux sélectionneurs de dinde, lesquels relèvent de deux groupes seulement par le jeu des holdings multi-filières. La confidentialité, voire le secret, y sont la règle, d'où des chiffres non officiels relatifs aux performances ou aux parts de marché, et une absence de chiffres concernant certaines données jugées stratégiques telles que les effectifs détenus par lignée en sélection. Ils peuvent être parfois très importants, comme le suggèrent les nombres de candidates contrôlées par génération dans les lignées ponte étudiées dans certaines publications (Besbes et al 1992, Ducrocq et al 2000). Quant aux tests comparatifs de produits commerciaux (chair, ponte) réalisés dans le passé en Europe et notamment en France, ils se sont progressivement réduits et ont fini par disparaitre.

La structuration des schémas de sélection et de croisement est toutefois assez classique. La figure 8 (EFSA 2010, d'après Hocking et McCorquodale 2008) représente les différents étages de la pyramide de sélection/ multiplication/croisement aboutissant typiquement à la production d'une «souche commerciale» de poulet de chair, en l'occurrence un produit terminal. Le plan de croisement met en jeu quatre populations sélectionnées, soit deux dans chacune des voies mâle et femelle. Cette figure met en évidence deux caractéristiques fortes des schémas avicoles : d'une part l'existence d'un étage intermédiaire de multiplication en race pure (produisant des «GGP», pour great grand parents, terme anglais utilisé usuellement) permettant de démultiplier considérable- ment les effectifs diffusés depuis l'étage de sélection; d'autre part, la réalisation des croisements dans un seul sens, avec élimination d'un sexe à chaque étape (à noter que dans le cas des pondeuses ou du canard mulard, ce processus de sexage est poursuivi jusqu'au produit terminal). Chaque lignée est donc sélectionnée pour occuper une place extrêmement précise dans le plan de croisement. Ainsi, la lignée voie femelle à l'origine des reproductrices utilisées lors du croisement produisant les femelles parentales est la plus sollicitée numériquement (et parfois qualifiée à ce titre de «lignée pivot») tandis qu'à l'opposé, la lignée voie mâle à l'origine des coqs utilisés lors du croisement produisant les coqs parentaux l'est très peu, comme illustré par la figure 8. Pour des raisons évidentes de maintien de la variabilité génétique, les effectifs entretenus en sélection dans les différentes lignées ne varient évidemment pas dans les mêmes proportions. On notera par ailleurs que dans le schéma présenté, le rapport d'effectifs détenus pour «descendre» d'un étage à l'autre dans la voie femelle est fixé forfaitairement à x 50 , à comparer avec la situation des schémas porcins, moins favorable.

Ce qui précède s'applique également aux sélectionneurs internationaux implantés (au moins pour partie) sur le territoire national. Toutefois, dans une certaine mesure, la France constitue une exception du fait de la diversification de ses productions avicoles, tant intraespèce qu'entre espèces. Les sélectionneurs avicoles y sont donc à la fois plus nombreux et plus divers quant à leur taille, l'ampleur des marchés auxquels ils sont adossés et les zones géographiques qu'ils desservent. Cette situation a incité la plupart d'entre eux à partager des outils de gestion génétique communs (au premier rang desquels la gestion informatique des données et l'évaluation génétique) à travers une structure unique, le SYSAF, devenu par la suite SYSAAF (Syndicat des Sélectionneurs Avicoles et Aquacoles Français). L'accès à d'autres services communs (certification des souches Label Rouge en particulier) a d'autre part conduit les opérateurs des filières standard, pourtant autonomes pour la gestion génétique de leurs lignées, à rejoindre également ce syndicat. L'inventaire des adhérents et des espèces sélectionnées est consultable sur le site du SYSAAF (http://www.sysaaf.fr). La liste des lignées et les effectifs détenus n'y sont pas mentionnés. Toutefois, le référentiel de sélection du syndicat (SYSAAF 2007) donne quelques repères. Ce référentiel est utilisé pour valider l'adhésion des nouveaux adhérents après période probatoire et pour la certification des souches Label Rouge. Dans 
Figure 8. Pyramide de sélection et de multiplication d'une lignée commerciale de poulets de chair, d'après EFSA, 2010.

\section{Generation}

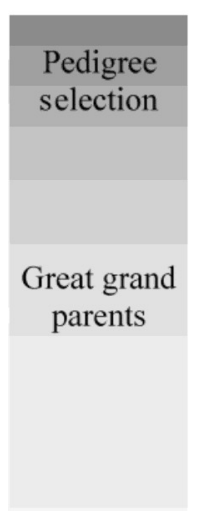

Grandparents
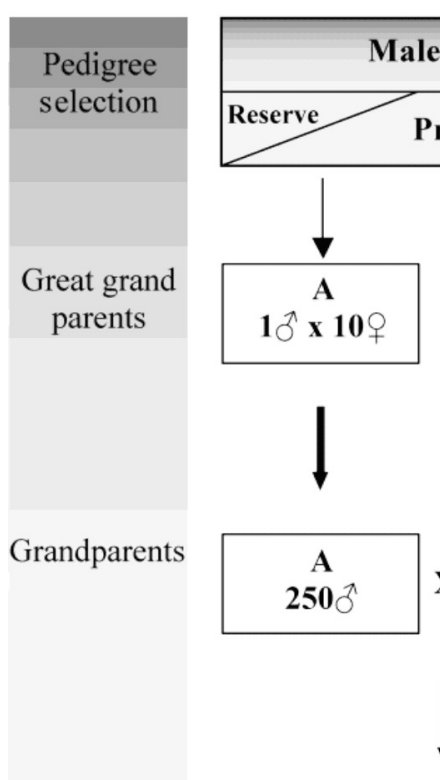

Parents

Hybrid

broilers
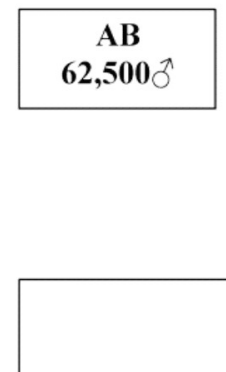

B

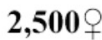

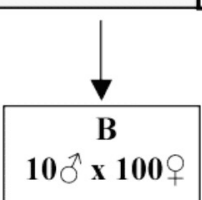

Conservation lines
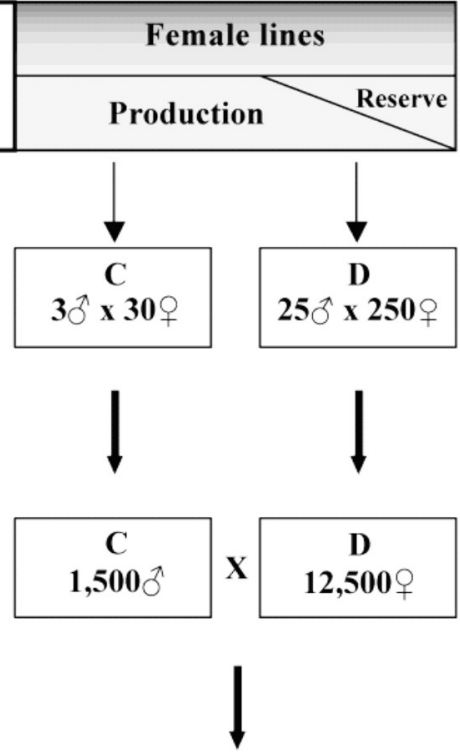

$\mathbf{X}$

CD

$\mathbf{6 2 5 , 0 0 0}$ 우
Genetic change

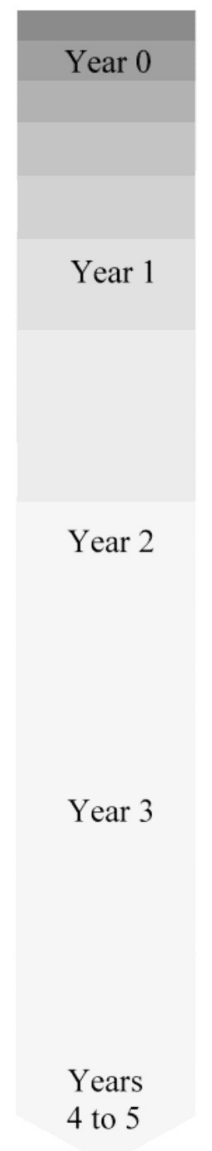

Les effectifs en encadré renvoient aux nombres relatifs de reproducteurs nécessaires pour engendrer les individus de la génération suivante, en référence à la valeur minimale $(n=1)$ affectée au sexe le moins limitant de la lignée la moins sollicitée numériquement.

En pratique, beaucoup plus d'individus contribuent au sommet de la pyramide, l'objectif recherché ici étant de représenter l'impact génétique des reproducteurs du noyau de sélection.

l'espèce Gallus gallus, par exemple, l'effectif minimum requis lors de chaque génération est de 40 pères, 200 mères et 2000 descendants contrôlés en lignée voie femelle, avec réduction de moitié en voie mâle. Ces effectifs sont parfois largement dépassés, en particulier dans le cas des lignées voie femelle dites «pivot».

Enfin, la segmentation intra-espèce des marchés est également à l'origine d'une autre spécificité des schémas de sélection et de croisement dédiés à la production de poulets colorés (plumage, peau, pattes...) : l'utilisation d'un coq terminal issu directement d'une unique lignée sélectionnée de façon à transmettre aux descendants un phénotype visible spécifique.

\section{3 / Ressemblances et dissem- blances}

\section{a) Esquisse de portraits-robots}

Par effet de contraste, les modalités d'organisation des schémas de sélection porcins et avicoles apparaissent très voisines comparées à celles des schémas de sélection mis en œuvre chez les ruminants. Trois portraits-robots sont esquissés au tableau 7, le bovin servant là encore de référence pour les filières de ruminants. En résumé :

- Le schéma pyramidal porc/volailles, conduisant à des produits terminaux «composites» issus de populations multiples, s'oppose à l'auto-renouvellement et à la production commerciale en race pure, très largement majoritaire chez les bovins ;

- Les différences observées dans la capacité de diffusion des reproducteurs sont plus nuancées et doivent s'analyser séparément selon les voies mâle et femelle. En voie mâle, elles résultent largement (quoique non exclusivement) de l'aptitude des espèces à la fourniture d'un nombre élevé de doses d'insémination, laquelle décroît très nettement du bovin aux volailles. En voie femelle, elles dépendent de la seule capacité reproductive et la tendance s'en trouve totalement inversée ;
- Les schémas pyramidaux sont désavantagés par leur délai de mise sur le marché des reproducteurs parentaux (et donc des terminaux), lié aux phases successives de multiplication et/ou de croisement permettant de «descendre les étages» de la pyramide. Ce délai n'existe pas en race pure, les reproducteurs devenant disponibles dès qu'ils sont matures sexuellement et évalués génétiquement (de manière désormais très précoce grâce à l'évaluation génomique chez les bovins laitiers) ;

- Dans les schémas pyramidaux, le concept dominant aux yeux des utilisateurs finaux est le type génétique, associé à un niveau génétique moyen attendu. En situation d'autorenouvellement au contraire, l'éleveur attache de l'importance au reproducteur considéré à titre individuel ;

- Les reproducteurs parentaux sont les seuls à être diffusés hors du schéma de sélection et croisement. Comme indiqué précédemment, dans le cas des mammifères cette diffusion concerne principalement les femelles, celle de la voie 
Tableau 7. Principales caractéristiques distinguant les schémas de sélection porcine et avicole de celui des bovins.

\begin{tabular}{|c|c|c|c|}
\hline & Bovin & Porc & Volailles \\
\hline $\begin{array}{l}\text { Auto-Renouvellement } \\
\text { vs schéma pyramidal }\end{array}$ & $\therefore$ & & \\
\hline $\begin{array}{l}\text { Produit de race pure } \\
\text { vs } \\
\text { croisement }^{1}\end{array}$ & & & $O D$ \\
\hline Diffusion $^{2}$ voies & $* * * * I^{*}$ & $* * * I^{* *}$ & $* * I^{* * *}$ \\
\hline $\begin{array}{l}\text { Délai de diffusion des } \\
\text { reproducteurs }\end{array}$ & $\begin{array}{c}\text { Sitôt } \\
\text { évaluation }\end{array}$ & $\begin{array}{c}\text { Délai de } \\
\text { production des } \\
\text { hybrides } \\
\text { parentaux }\end{array}$ & $\begin{array}{c}\text { Délai de } \\
\text { production des } \\
\text { hybrides } \\
\text { parentaux }\end{array}$ \\
\hline Concept dominant & Reproducteur & Type génétique & Type génétique \\
\hline Prix $^{2}$ des reproducteurs & $* * *$ & ** & * \\
\hline $\begin{array}{l}\text { Propriété des } \\
\text { races/lignées }\end{array}$ & Collective & $\begin{array}{c}\text { Collective / } \\
\text { Privée }\end{array}$ & Privée \\
\hline $\begin{array}{l}\text { Mise en œuvre de la } \\
\text { sélection }\end{array}$ & Répartie & +- Autonome & Autonome \\
\hline Maîtrise du croisement & - & Autonome & Autonome \\
\hline Marché & National & National / Mondial & Mondial / National \\
\hline
\end{tabular}

1 Les secteurs colorés représentent les contributions relatives des races ou lignées à l'origine des produits terminaux (exclusive vs «composite»; dans le cas des volailles, le petit cercle disjoint rappelle l'existence du croisement inter-générique à l'origine du canard mulard).

2 Ordres de grandeur relatifs estimés grossièrement.

mâle étant réalisée majoritairement via la semence. Le prix moyen des reproducteurs parentaux reste étroitement lié au format de l'espèce et décroit très fortement du bovin aux volailles. Cette dépendance à la valeur du produit commercial correspondant peut être considérée comme un handicap pour les sélectionneurs des espèces de moindre gabarit. Il est en effet difficile de répercuter des coûts de sélection élevés sur le prix de vente des reproducteurs, quand bien même ils induiraient une plusvalue considérable au niveau de la filière via la nombreuse descendance qu'ils engendrent (cette observation vaut particulièrement pour les reproducteurs de la voie mâle en filière avicole, diffusés en relativement faible nombre);

- La propriété des populations évolue selon un gradient allant du collectif (à travers des organisations raciales) chez le bovin au privatif chez les volailles, en passant par une situation intermédiaire propre à la sélection porcine, où coexistent les deux types d'opérateurs (avec prééminence du collectif dans le cas de la France, ce qui n'est pas une règle générale);

- La mise en œuvre de la sélection suit également un gradient de ce type. Chez les bovins, les différentes étapes (procréation, gestion généalogique, contrôle des performances, centralisation des données, évaluation génétique ...) sont réalisées par des opérateurs distincts, exerçant des métiers complémentaires au sein d'une chaîne. Cette situation contraste fortement avec celles des schémas avicoles, pour lesquels l'ensemble des étapes est réalisé en autonomie par un opérateur unique, le sélectionneur. La situation porcine est là encore intermédiaire, quoique globalement plus proche de celle des volailles. D'une part, certains de ses opérateurs sont autonomes. D'autre part, les acteurs du dispositif collectif disposent d'une grande latitude quant à la gestion des cheptels de sélection qu'ils détiennent, malgré l'orientation collective de ces races et le recours à des infrastructures (stations publiques de contrôle de performances) où à des outils (centralisation des données, évaluation) de nature collective ;

- Au stade du plan de croisement (qui ne concerne que les schémas pyramidaux), la mise en œuvre par les opérateurs génétiques peut être considérée comme totalement autonome, y compris dans le cas du porc ;

- Enfin, l'extension géographique du marché des reproducteurs évolue selon un gradient croissant allant du national dans le cas des bovins (marché de la semence excepté) à l'international prédominant (explicité plus loin) dans le cas des volailles. La situation du porc est à nouveau intermédiaire, la part de marché détenue par des opérateurs nationaux restant fréquemment prépondérante.

Ainsi, considérées par rapport à la situation des bovins, les différences entre schémas porcins et avicoles relèvent moins $\mathrm{du}$ fond que de relatives nuances, ces dernières résultant d'effets d'échelle liés notamment aux différen- ces de gabarit et de taux de reproduction entre porcs et volailles. Ces similitudes ont amené quelques opérateurs génétiques internationaux à se positionner simultanément sur les marchés de la génétique porcine et avicole.

b) Quelques particularités influençant la typologie des opérateurs

Pour autant, le schéma pyramidal «porc/volailles» n'est pas monolithique.

Certaines différences biologiques entre porc et volailles confèrent à ces dernières un avantage significatif. C'est le cas en particulier de leur gabarit modeste qui, en réduisant l'espace nécessaire et les coûts d'entretien, facilite le regroupement des lignées en un même lieu (sauf duplicata constitués à titre de sécurité sanitaire). Il n'est pas rare que plusieurs milliers de reproducteurs et leur suite soient hébergés sur un même site. Le faible encombrement des oisillons, conjugué à l'autonomie alimentaire conférée par leur vésicule vitelline, autorise le transport à longue distance d'effectifs importants, permettant le réapprovisionnement régulier de cheptels «GGP» dans différentes régions du monde. Ce faible encombrement permet également l'élimination précoce des rebuts de sexage, dans le respect de la réglementation prévue à cet effet. En comparaison, les noyaux de sélection porcins sont beaucoup moins centralisés, souvent répartis sur de nombreux sites (cas des dispositifs collectifs), et se prêtent mal à une diffusion massive à longue distance permettant de déployer aisément un schéma génétique au niveau mondial. Du fait d'un taux de reproduction moindre, l'étage de multiplication requiert également la mise en place de cheptels dédiés en plus grand nombre. Enfin, la nature des utilisateurs des reproducteurs parentaux, et donc des clients finaux, est également différente. L'accouveur est lui aussi un industriel, en capacité de traiter des volumes importants et constituant un point d'accès commode à la filière de production, tant chair que ponte. Dans le cas du porc, il s'agit d'éleveurs naisseurs, lesquels constituent une clientèle dispersée. Pour y accéder et assurer la logistique de livraison afférente, des structures relais, telles que les groupements de producteurs dans le cas de la France, constituent donc souvent des partenaires incontournables.

La segmentation des marchés constitue également un facteur de différenciation majeur entre opérateurs de sélection des filières porcine et avicole.

La filière porcine est très peu segmentée, la part de marché des productions sous signe de qualité (Label Rouge, cer- 
Tableau 8. Segmentation des marchés en filière avicole pour la production de chair et d'œuf.

\begin{tabular}{|c|c|c|c|c|}
\hline \multirow{2}{*}{$\begin{array}{l}\text { Type de } \\
\text { marché }\end{array}$} & \multicolumn{2}{|r|}{ Chair } & \multicolumn{2}{|c|}{ CEuf } \\
\hline & Intra espèce & Entre espèces* & Intra espèce ${ }^{5}$ & Entre espèces* \\
\hline $\begin{array}{l}\text { Marchés } \\
\text { de masse } \\
\text { (monde) }\end{array}$ & $\begin{array}{l}\text { Produits } \\
\text { standards à } \\
\text { segmentation } \\
\text { limitée }^{* *}\end{array}$ & $\begin{array}{l}\text { Poulet } \\
\text { Dinde } \\
\text { Canard commun }\end{array}$ & $\begin{array}{l}\text { CEuf standard } \\
\text { brun } \\
\text { CEuf standard } \\
\text { blanc }\end{array}$ & $\begin{array}{l}\text { Poule } \\
\text { Cane commune } \\
\text { (en Asie) }\end{array}$ \\
\hline $\begin{array}{l}\text { Marchés } \\
\text { de niche } \\
\text { (cas de la } \\
\text { France) }\end{array}$ & $\begin{array}{l}\text { Label Rouge } \\
\text { Certifié }^{1} * * * \\
\text { Bio }^{2} \\
\text { AOC }^{3} \\
\text { Races locales }\end{array}$ & $\begin{array}{l}\text { Canard de Barbarie } \\
\text { Canard mulard (foie } \\
\text { gras) } \\
\text { Pintade } \\
\text { Oie (chair / foie gras) } \\
\text { Caille } \\
\text { Pigeon }\end{array}$ & $\begin{array}{l}\text { Label Rouge } \\
\text { Certifié } \\
\text { Bio } \\
\text { Races locales }\end{array}$ & Caille \\
\hline
\end{tabular}

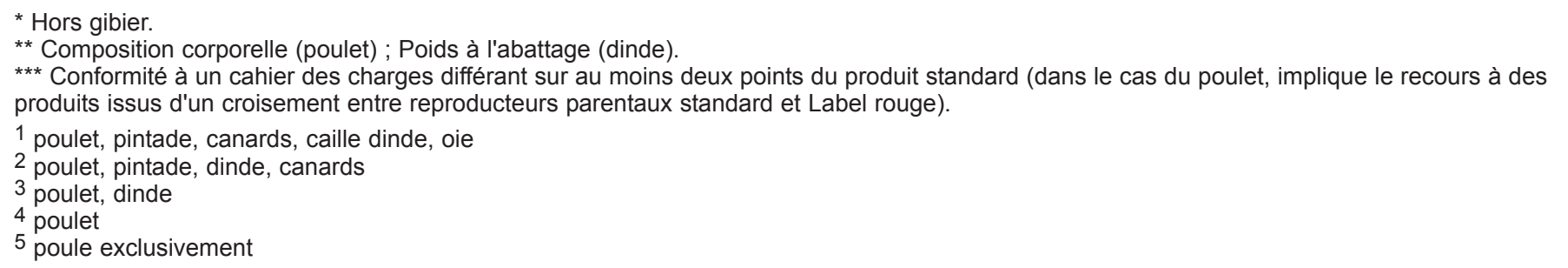

tifié ou Bio) y étant très faible. Les types génétiques utilisés pour ces dernières sont peu différents et ne donnent pas lieu à la sélection de races ou lignées spécifiques (hormis dans le cas des productions issues de races rustiques, lesquelles demeurent anecdotiques). Au niveau des produits standards, seule une très relative segmentation est observée, certains pays recourant en voie mâle à des types génétiques porteurs de l'allèle de la sensibilité à l'halothane et d'autres pas. Aussi, la typologie des opérateurs génétiques ne s'en trouve nullement affectée.

En filière avicole, au contraire, la segmentation est particulièrement importante, tant entre espèces qu'intraespèce, comme illustré au tableau 8 pour la chair et l'œuf respectivement. Entre espèces, les marchés mondiaux de masse se répartissent entre trois espèces majeures pour la chair (poulet, dinde et canard commun) et deux pour la ponte (poule et en Asie cane pondeuse). Les marchés de niche, quant à eux, recouvrent un nombre bien supérieur d'espèces, en particulier en France pour la chair (canard de Barbarie, canard mulard, pintade, oie, caille et pigeon) et dans une bien moindre mesure pour la ponte (caille). Intraespèce, la segmentation est également importante. Elle concerne peu les marchés de masse (hormis le cas particulier de la consommation d'œufs blancs ou bruns, liée aux habitudes de consommation nationales) mais prioritairement les marchés de niche, dont la part de marché est très significative en France en particulier (Label Rouge, certifié, Bio, AOC et races locales), et ce pour de nombreuses espèces, du moins en chair. Chez le poulet en particulier, elle a conduit au développement de souches à croissance lente ou au maintien de l'utilisation de races anciennes. Ainsi, à poids vif quasi égal, l'âge d'abattage des poulets standard, certifié (croisement standard x Label Rouge), Label Rouge ou AOC Bresse est de l'ordre de 40, 56, 84 ou 110 jours, respectivement. Pour plus de détails sur les produits avicoles sous signe de qualité, consulter les sites du Syndicat National des Labels Avicoles de France (http://synalaf.com) et de l'Association de Promotion de la Volaille Française (http://www.volaille-francaise.fr).

Au niveau des marchés de masse, la segmentation entre espèces (ainsi qu'entre filières chair et ponte) avait conduit dans le passé à l'émergence de sélectionneurs dédiés. Toutefois, la tendance des dernières années est clairement au regroupement, avec la constitution de sociétés holding opérant sur différents marchés à la fois. Au niveau des marchés de niche, comme évoqué précédemment au plan national, la segmentation tant entre espèces (multiples) qu'intra-espèce (produits standard vs Label Rouge notamment) a favorisé l'émergence d'opérateurs de sélection beaucoup plus diversifiés. Certains sélectionneurs se sont néanmoins positionnés d'emblée sur les deux segments des marchés de masse et de niche. Les regroupements via des sociétés holding sont de nature à renforcer cette tendance.

\section{6 / Nouvelles contraintes, nouvelles opportunités?}

\section{1 / La montée des demandes sociétales}

a) Réglementation relative au bienêtre animal

Le secteur de la génétique animale est de plus en plus concerné par la montée des demandes sociétales concernant l'élevage (Dockès et al 2011). Ces demandes peuvent conduire à deux types d'évolutions, non exclusives l'une de l'autre : d'une part, une modification de la demande des consommateurs, incitant à recourir à des modes d'élevage ou des objectifs de sélection différents ; d'autre part, l'adoption de contraintes réglementaires sous la pression d'associations de protection animale, conduisant à l'interdiction de certaines pratiques d'élevage ou de la poursuite de certains objectifs de sélection. En pratique, c'est l'évolution du corpus réglementaire dans le cadre de l'Union Européenne qui a eu l'impact le plus déterminant sur les conditions d'élevage des animaux de rente, en particulier à travers la réglementation relative au bien-être animal. Toutes les filières animales sont visées par la Directive «socle» 98/58/CE du 20 juillet 1998 concernant la protection des animaux dans les élevages, laquelle pose notamment le principe que tout animal doit bénéficier d'un logement, d'une alimentation et de soins appropriés à ses besoins physiologiques et éthologiques. Toutefois, les filières avicole et porcine 
ont fait précocement l'objet d'une attention particulière, avec des directives spécifiques déjà abrogées et remplacées pour certaines d'entre elles (poules pondeuses, porcs).

A ce jour, les opérateurs génétiques ne sont pas spécifiquement impliqués, les directives en vigueur (transposées en droit national) étant focalisées sur les conditions d'élevage. La directive 2008/120/CE du 18 décembre 2008 établissant les normes minimales relatives à la protection des porcs n'introduit aucune disposition spécifique aux cheptels de sélection et multiplication, pour lesquels les dispositions prévues ne posent pas de problème spécifique d'application. La situation des cheptels de sélection et multiplication avicoles est plus délicate, dans la mesure où certaines particularités de leur gestion (élevage en cage individuelle des reproducteurs, rationnement forcé des reproducteurs de souches lourdes, élimination d'un des sexes à l'éclosion) posent des problèmes d'acceptabilité. La Directive 1999/74/CE du 19 juillet 1999 établissant les normes minimales relatives à la protection des poules pondeuses ne s'applique pas aux cheptels reproducteurs (lesquels restent néanmoins soumis aux exigences pertinentes de la directive 98/58/CE), pas plus que la directive 2007/43/CE du 28 juin 2007 fixant des règles minimales relatives à la protection des poulets destinés à la production de viande. En revanche, cette dernière directive marque un tournant, puisqu'elle précise dans ses considérants qu'il convient que la Commission présente un rapport prenant en considération d'autres recherches et l'expérience pratique afin d'améliorer encore le bien-être des poulets destinés à la production de viande, y compris le bien-être des cheptels parentaux de ces poulets. Une évolution ultérieure, plus contraignante, n'est donc pas à exclure.

Les directives précitées ne visent pas expressément la possibilité ou non de poursuivre certains des objectifs de sélection actuels. Toutefois, la directive «poulets de chair» marque là encore un tournant, puisqu'il est également précisé dans ses considérants que le rapport à présenter par la Commission devra en outre examiner des possibilités d'établir des seuils pour les signes de carences en matière de bien-être détectés pendant l'inspection post mortem ainsi que l'influence des paramètres $(N B$ : au sens de facteurs) génétiques sur les irrégularités constatées. Or, à la demande de la Commission, un avis scientifique a été rendu récemment (EFSA 2010) par le Panel EFSA Santé et Bien-être Animal (AHAW) sur l'influence des facteurs génétiques sur le bien-être et la résistance au stress des poulets de chair com- merciaux. Il met en exergue plusieurs effets préjudiciables sur les produits commerciaux (problèmes d'aplombs, pododermatites, ascites, syndrome de mort subite) attribués à une sélection intense et continue de la vitesse de croissance, de l'efficacité alimentaire et de la conformation corporelle, jugée inappropriée. L'avis recommande notamment de contre-sélectionner ces défauts (après objectivation, établissement de grilles de notation standardisées d'un pays à l'autre et collecte de données, y compris commerciales), de sélectionner en faveur de la mobilité et de l'activité des animaux, de mieux adapter les types génétiques aux conditions de milieu (croissance réduite en milieu chaud), de maintenir la variabilité génétique, et d'utiliser des outils tels que la sélection génomique et d'autres nouvelles technologies pour sélectionner les critères de bien-être animal. La prise en compte de cet avis par la Commission constituerait donc un premier pas vers une réglementation relative aux objectifs de sélection euxmêmes, appliquée au cas du poulet de chair. A terme, il pourrait en aller de même pour d'autres effets préjudiciables posant des problèmes d'acceptabilité chez les pondeuses (fractures osseuses...) ou chez le porc (susceptibilité au syndrome de stress chez les porteurs homozygotes de l'allèle $n$, problèmes d'aplombs, surmortalité des porcelets...).

On notera par ailleurs que les avis scientifiques rendus par l'EFSA s'attachent principalement à aider les gestionnaires de risques à identifier des méthodes pour réduire toute condition inutile de douleur, de détresse et de souffrance pour les animaux, ainsi qu'à accroître leur bien-être chaque fois que cela est possible. En revanche, il n'est pas du ressort de l'EFSA de formuler un avis sur les questions éthiques ou culturelles liées au bien-être des animaux. Or, les considérations éthiques peuvent parfois entrer en conflit avec les possibilités techniques et/ou économiques. Les opérateurs génétiques des filières avicole et porcine doivent donc se montrer particulièrement attentifs aux demandes sociétales en matière d'élevage et plus particulièrement de bien-être animal, toujours susceptibles de déboucher sur de nouvelles contraintes réglementaires.

\section{b) Le projet Code-EFABAR}

C'est pourquoi, dès le début des années 2000, l'EFFAB (European Forum of Farm Animal Breeders), qui réunit de nombreux opérateurs de la sélection et de la reproduction animale toutes espèces confondues, a coordonné le projet SEFABAR (Sustainable
European Farm Animal Breeding And Reproduction) financé par l'Union Européenne (Liinamo et Neeteson-van Nieuwenhoven 2003). Au cours de ce projet, dont le périmètre excédait le seul bien-être animal, les acteurs concernés, incluant des représentants des firmes de sélection, des ONG (dont associations de protection animale) et des universitaires spécialistes en économie, sociologie et éthique, ont collaboré pour identifier des priorités en vue d'une sélection et d'une reproduction jugées plus durables. Ces résultats ont ensuite été utilisés dans le cadre d'un autre projet, Code-EFABAR (Code of Good Practice for European Farm Animal Breeding and Reproduction), également financé par l'Union Européenne, dont le but était de développer un code de bonnes pratiques pour le secteur de la sélection et de la reproduction animales. Ce code, mis en place à compter de 2006, comporte trois sections : principes généraux (dont le principe d'une sélection dont les effets sont équilibrés) ; durabilité, déclinée en six critères (sécurité alimentaire, qualité des produits, diversité génétique, efficacité, environnement, santé et bien-être animal) ; technologies (génétique et reproduction). Ces deux dernières sections comportent des recommandations mais fournissent également des informations sur la façon dont les sélectionneurs satisfont ces critères. A ce jour, une douzaine d'opérateurs ont opté pour ce code, plus de la moitié d'entre eux opérant dans les filières porcine et surtout avicole (la part de marché représentée par ces derniers étant prééminente). Les exemples figurant dans le guide de mise en œuvre disponible sur le site de l'EFFAB (www.code-efabar.org) suggèrent que les recommandations formulées vont dans le bon sens, mais sans remettre profondément en cause les critères et méthodes de sélection ou les pratiques d'élevage actuelles. L'exemple fourni en filière avicole reprend plusieurs des recommandations formulées par le panel AHAW dans le cas du poulet de chair. Toutefois, aucune solution techniquement et économiquement viable n'est encore disponible, donc proposée, sur le point crucial de l'élimination des poussins suite au sexage.

\section{2 / La sélection génomique}

Les nouvelles technologies, en particulier la sélection génomique, offrent toutefois de nouvelles opportunités, comme souligné dans l'avis du panel EFSA à propos des critères de bien-être animal. Avec l'irruption attendue de cette nouvelle méthode de sélection, on peut se demander si des changements majeurs sont en passe d'intervenir, comme constaté récemment chez les bovins laitiers. En fait, chez le porc et 
les volailles, des évolutions semblent plus probables qu'une révolution. Le principe même du schéma pyramidal de sélection et croisement ne peut être remis en cause, compte tenu de ses nombreux avantages. Les conditions du contrôle de performances, en revanche, devraient être significativement modifiées, puisqu'elles concerneront des animaux parentaux ou terminaux plutôt que des populations pures, comme illustré dans l'exemple de mise en œuvre de la sélection génomique chez le porc présenté dans ce même numéro (Tribout 2011).

Chez le porc et les volailles, où l'intervalle moyen entre générations est déjà court, des gains de progrès génétique annuel sont attendus sur les caractères mesurés classiquement mais tardivement sur les femelles (prolificité, ponte...) plutôt que sur les caractères précoces classiques aisément mesurables, généralement dans les deux sexes (croissance, composition corporelle...). Toutefois, c'est la prise en compte de caractères nouveaux (mesures fines de qualité du produit, par exemple) et/ou non mesurables en routine (en milieu confiné pour des tests de résistance à des agents pathogènes, par exemple) qui devrait constituer la principale innovation, en s'appuyant notamment sur de nouvelles technologies (biocapteurs, imagerie...). Ainsi, la capacité à phénotyper finement des populations de référence constitue un enjeu fort. Pour autant, et contrairement aux caractères mesurés en routine en élevage, leur coût d'acquisition peut être élevé, ce qui constitue un premier frein.

Le second tient au coût de génotypage des populations de référence, dont la taille est inversement proportionnelle à l'héritabilité des caractères. Un autre moyen permettant d'accroître la précision des phénotypes (caractères tardifs peu héritables, en particulier), et donc de limiter la taille des populations de référence consisterait à prendre en compte non pas la performance des individus mais la valeur moyenne des descendants des reproducteurs génotypés (nombreux dans ces filières, $\mathrm{y}$ compris en voie femelle), comme c'est le cas dans l'évaluation génomique bovine laitière. Toutefois, elle n'est applicable qu'aux caractères déjà mesurés en routine dans les élevages. Une autre possibilité (Tribout 2011) consiste à «mutualiser» les populations de référence par la constitution de panels multi-types génétiques raisonnablement proches, ce qui permet de réduire globalement le nombre d'individus génotypés, tout en partageant les coûts de phénotypage dès lors qu'il s'agit de nouveaux caractères nécessitant des mesures spécifiques.

Le dernier frein en termes de coût, et non le moindre, tient au nombre de candidats qu'il conviendrait de génotyper pour générer un progrès génétique annuel au moins équivalent à celui réalisé actuellement sur les caractères classiques. La situation est évidemment différente avec les nouveaux caractères, pour lesquels le gain de progrès génétique sera net. Pour autant, seule une réduction drastique des coûts de génotypage, du fait de grands volumes couplés à des méthodologies alternatives (puces basse densité et méthodes d'imputation, par exemple), apparaît compatible avec un développement significatif de la sélection génomique chez le porc et les volailles ; car malgré l'ampleur de la diffusion de reproducteurs dans ces filières, un surcoût par trop important en regard de la valeur intrinsèque de l'animal «support» de progrès génétique semble aujourd'hui problématique, en particulier en filière avicole. Les sélectionneurs avicoles bénéficieraient en revanche d'une retombée, la réassignation a postériori des produits à leurs parents, permettant de s'affranchir de la traçabilité des œufs pondus et donc de recourir à une reproduction en groupes, moins contraignante et résolvant de surcroît le problème d'acceptabilité sociale des cages individuelles.

Plus généralement, la question posée est celle des moyens financiers à mobiliser pour mettre en œuvre la sélection génomique dans les filières porcine et avicole. Un retour aux opérateurs génétiques sera indispensable pour supporter les surcoûts d'une sélection à la fois plus efficace et plus équilibrée, qui bénéficiera non seulement aux utilisateurs directs (éleveurs) mais aussi à l'aval des filières, jusqu'aux consommateurs voire aux citoyens (critères de bien-être animal, par exemple). Les modalités de ce retour pourraient éventuellement différer selon le type de sélection mis en œuvre par les opérateurs : la sélection collective (porc) repose sur un fondement coopératif et s'inscrit dans une logique verticale de filière, tandis que la sélection autonome (porc, volailles) relève de l'initiative et de capitaux privés et suit une logique de marché des reproducteurs, horizontale et multi-filières. La «mutualisation» évoquée précédemment permettrait de réduire les coûts. Elle peut aller jusqu'à la mise en commun des données de génotypage et de phénotypage obtenues séparément par différents opérateurs, de façon à améliorer la précision des valeurs génomiques prédites et donc l'efficacité globale des schémas. De telles approches semblent compatibles avec la tradition de collaboration des opérateurs de la sélection collective porcine. Elles le sont moins dans le contexte des opérateurs privés, porcins et surtout avicoles, pour lesquels la concurrence et le secret sont à ce jour la règle.

\section{Conclusion}

Les particularités biologiques propres au porc et aux volailles ont conduit les opérateurs génétiques à opter pour un mode d'organisation de leurs schémas de sélection et croisement de type pyramidal. Ces modalités ont en quelque sorte façonné les opérateurs génétiques eux-mêmes, par delà les différences induites par certaines particularités biologiques ou économiques propres à ces espèces ou filières, de même que par l'importance relative des secteurs privé et collectif. La mise en œuvre de la sélection génomique ne devrait pas bouleverser cette typologie, les opérateurs détenant les races et/ou lignées sélectionnées, de manière souvent exclusive. Dans ce contexte, l'irruption d'opérateurs intervenant seulement sur une partie de la chaîne, pour la prédiction de la valeur génomique notamment, apparaît peu probable. Par contre, le renchérissement du coût de la sélection ainsi pratiquée pose des problèmes de faisabilité au plan économique, du fait notamment du nombre considérable de candidats à génotyper. La «mutualisation» de populations de référence, voire la mise en commun des données obtenues sur des populations de référence distinctes, constituent des solutions pour réduire les coûts et accroître l'efficacité globale des schémas. A défaut, la concentration pourrait encore se renforcer parmi les sélectionneurs de ces filières.

\section{Remerciements}

L'auteur remercie particulièrement Pierre Sellier pour ses conseils, son aide dans le choix et la mise à disposition des sources et sa lecture critique du manuscrit. 


\section{Références}

Agence de la Sélection Porcine, 2011. Répartition des élevages et des truies aux étages sélection et multiplication au 31/12/2007; http://www.asp.asso.fr/genetic/ genet j.htm ; Effectifs de truies productives en sélection au 31/12/2007 par catégorie de populations sélectionnées; http://www.asp.asso.fr /genetic/genet j.htm; Productivité numérique des truies parentales par organisation de sélection porcine - Exercice 2006. http:/ /www.asp.asso.fr/autres\%20pages/tests/tests $\mathrm{j}$. htm. Site consulté en septembre 2011.

Baéza E., Rideau N., Chartrin P., Davail S. Hoo-Paris R., Mourot J., Guy G., Bernadet M.D., Hermier D., 2004. Canards de Barbarie, Pékin et leurs croisements : aptitude à l'engraissement. 6èmes Journ. Rech. Palmipèdes à Foie Gras, Arcachon, France, 41-48.

Besbes B., Ducrocq V., Foulley J.L., Protais M., Tavernier A., Tixier-Boichard M. Beaumont C., 1992. Estimation of genetic parameters of egg production traits of laying hens by restricted maximum likelihood applied to a multiple-trait reduced animal model. Genet. Sel. Evol., 24, 539-552.

Bidanel J.P., 2011. Biology and genetics of reproduction. In: The genetics of the pig, $2^{\text {nd }}$ Edition, Rothschild M.F., Ruvinsky A., (Eds). CAB International, 218-241.

Bidanel J.P., Ducos A., Guéblez R., Labroue F., 1994. Genetic parameters of backfat thickness, age at $100 \mathrm{~kg}$ and ultimate $\mathrm{pH}$ in on-farm tested French Landrace and Large White pigs. Livest. Prod. Sci., 40, 291-301.

Chapuis H., Tixier-Boichard M., Delabrosse Y., Ducrocq V., 1996. Multivariate restricted maximum likelihood estimation of genetic parameters for production traits in three selected turkey strains. Genet. Sel. Evol., 28, 299-317.

Coquerelle G., 2000. Les poules, diversité génétique visible. Collection «Du labo au terrain», INRA éditions, Paris, France, 184p.

Directive 98/58/CE du Conseil du 20 juillet 1998 concernant la protection des animaux dans les élevages. Journal officiel de l'Union européenne $n^{\circ}$ L 221 du 08/08/1998, p. 0023 0027.

Directive 1999/74/CE du Conseil du 19 juillet 1999 établissant les normes minimales relatives à la protection des poules pondeuses. Journal officiel de l'Union européenne n L 203 du 03/08/1999, 0053-0057.
Directive 2007/43/CE du Conseil du 28 juin 2007 fixant des règles minimales relatives à la protection des poulets destinés à la production de viande. Journal officiel de l'Union européenne $n^{\circ}$ L 182 du 12/07/2007, 0019-0028.

Directive 2008/120/CE du Conseil du 18 décembre 2008 établissant les normes minimales relatives à la protection des porcs. Journa officiel de l'Union européenne $\mathrm{n}^{\circ} \mathrm{L} 47 \mathrm{du}$ 18/02/2009, 0005-0013.

Dockès A.C., Magdelaine P., Daridan D. Guillaumin A., Rémondet M., Selmi A. Gilbert H., Mignon-Grasteau S., Phocas F., 2011. Attentes en matière d'élevage des acteurs de la sélection animale, des filières de l'agroalimentaire et des associations. In : Numéro spécial, Amélioration génétique. Mulsant P. Bodin L., Coudurier B., Deretz S., Le Roy P., Quillet E., Perez J.M. (Eds). INRA Prod. Anim., 24, 285-296.

Ducos A., Bidanel J.P., 1996. Corrélations génétiques entre caractères de production et de reproduction mesurés en élevage dans les races porcines Large White et Landrace Français Journ. Rech. Porcine Fr., 28, 15-22.

Ducrocq V., Besbes B., Protais M., 2000. Genetic improvement of laying hens viability using survival analysis. Genet. Sel. Evol., 32 23-40

EFSA Panel on Animal Health and Welfare 2010. Scientific opinion on the influence of genetic parameters on the welfare and the resistance to stress of commercial broilers. EFSA J 8 (7):1666. 82p. http://www.efsa.europa.eu/ fr/scdocs/doc/1666.pdf

Hocking P.M., McCorquodale C.C., 2008 Similar improvements in reproductive performance of male line, female line and paren stock broiler breeders genetically selected in the UK and in South America. Brit. Poult. Sci. 49, 282-289.

IFIP, 2008. Le porc par les chiffres 2008. Ed. IFIP, 52p.

IFIP, 2009. Résultats du 29ème test de contrôle des produits terminaux issus de différentes combinaisons de types génétiques de truies et de verrats parentaux agréés par le Ministère chargé de l'Agriculture. TechniPorc, 32, 3-16.

Legault C., 1998. Génétique et prolificité chez la truie : la voie hyperprolifique et la voie sinoeuropéenne. INRA Prod. Anim., 11, 214-218.
Liinamo A.E. Neeteson-van Nieuwenhoven A.M., 2003. SEFABAR: Sustainable European Farm Animal Breeding And Reproduction. Proc. Final SEFABAR workshop, Rome, Italy, 4 September, AnNe Publishers. 123p.

Mérat P., 1984. The sex-linked dwarf gene in the broiler chicken industry. World's Poultry Sci., 40, 10-18.

Ollivier L., 1974. Optimum replacement rates in animal breeding. Anim. Prod., 19, $257-$ 271.

Sellier P., 1998. Major genes and crossbreeding with reference to pork quality. Polish J. Food Nutr. Sci., suppl. 7/48, 77-89.

Sellier P., 2010. Vers une utilisation accrue de l'hétérosis en amélioration des animaux ? Académie d'Agriculture de France, séance du 31 mars 2010. http://www.academie-agriculture.fr/detail-seance 224.html

SYSAAF, 2007. Référentiel : Mode de sélection des lignées et de production de reproducteurs parentaux avicoles. RefAvi SYSAAFRéférence 07.1, Version 01/02/2007, 38p.

Tribout T., 2011. Perspectives d'application de la sélection génomique dans les schémas d'amélioration génétique porcins. In : Numéro spécial, Amélioration génétique. Mulsant P., Bodin L., Coudurier B., Deretz S., Le Roy P. Quillet E., Perez J.M. (Eds). INRA Prod. Anim., 24, 369-376.

Tribout T., Bidanel J.P., 2008. Paramètres génétiques du nombre de porcelets nés vivants par portée et relations avec les caractères du contrôle en ferme dans les populations porcines Large White type femelle et Landrace Français. Journ. Rech. Porcine Fr., 40, 113-118.

Tribout T., Garreau H., Bidanel J.P., 1996. Paramètres génétiques de quelques caractères de qualité de la viande dans les races porcines Large White et Landrace Français. Journ. Rech. Porcine Fr., 28, 31-38.

Tribout T., Bidanel J.P., Garreau H., Flého J.Y., Guéblez R., Le Tiran M.H., Ligonesche B., Lorent P., Ducos A., 1998. Présentation du dispositif collectif français d'évaluation génétique porcin pour les caractères de production et de reproduction. Journ. Rech. Porcine Fr., $30,95-100$

\section{Résumé}

L'organisation des schémas de sélection découle des particularités biologiques (prolificité, intervalle de générations...) propres à chaque espèce ou groupe d'espèces, ainsi que de la nature des caractères d'intérêt (précoces, tardifs...). Dans les filières porcine et avicole, où la diffusion des reproducteurs est largement mondialisée, la concurrence entre opérateurs induit une course au progrès génétique impliquant de maximiser l'efficacité de la sélection dans les races ou lignées pures, ainsi que de profiter des différents avantages du croisement (hétérosis, complémentarité entre populations, utilisation de gènes majeurs) dans les limites permises par le taux de reproduction élevé de ces espèces. La mise en ouvre de ces principes conduit à des modalités d'organisation des schémas de sélection et de croisement de type pyramidal, caractéristique de ces filières. Elles exercent en retour un effet structurant sur les opérateurs génétiques eux-mêmes, conduisant à une typologie caractéristique, modulée par certaines particularités biologiques des animaux (format...), par la segmentation du marché des produits commerciaux dans la filière avicole (espèces, marchés de niche...), et par l'importance relative du secteur privé (partielle en filière porcine, totale en filière avicole). L'arrivée de la sélection génomique ne devrait pas bouleverser cette typologie, mais pose des problèmes de faisabilité économique. 


\begin{abstract}
Constraints and opportunities for the organization of selection in porcine and poultry production

The organization of selection schemes results from biological specificities (prolificacy, generation intervals...) of each species along with the nature of the traits (early, late...). In porcine and poultry production where the distribution of breeding animals is global, the competition between operators has induced a race towards genetic progress implicating maximizing selection efficiency in pure breeds or lines, as well as taking advantage of crosses (heterosis, complementarity between populations, use of major genes) within the limits allowed by the high reproduction rate of these species. The use of these principles has led to the typical organization of selection schemes and pyramidal crossing, both characteristics of these productions. This organization structures the genetic operators themselves, leading to a characterized typology, modulated by biological specificities of the animals (size...), by the segmentation of commercial poultry product markets (species, niche markets...), and by the relative importance of private property of breeding animals (partial in pigs, total in poultry). The occurrence of genomic selection should not upset this typology, but creates problems of economic feasibility.
\end{abstract}

COUDURIER B., 2011. Contraintes et opportunités d'organisation de la sélection dans les filières porcine et avicole. In : Numéro spécial, Amélioration génétique. Mulsant P., Bodin L., Coudurier B., Deretz S., Le Roy P., Quillet E., Perez J.M. (Eds). INRA Prod. Anim., 24, 307-322. 
